\title{
The capture of main sequence stars and giant stars by a massive black hole
}

\author{
Y. Lu, ${ }^{1}$ Y. F Huang, ${ }^{2}$ Z. Zheng ${ }^{1}$ and S. N. Zhang ${ }^{3}$ \\ ${ }^{1}$ National Astronomical Observatories, Chinese Academy of Sciences, Beijing 100012, China \\ ${ }^{2}$ Department of Astronomy, Nanjing University, Nanjing 210093, China \\ ${ }^{3}$ Physics Department and Center for Astrophysics, Tsinghua University, Beijing 100084, China \\ email: ly@bao.ac.cn;
}

\begin{abstract}
Since the mass-radius relation is quite different for a main sequence (MS) star and a giant $(\mathrm{G})$ star, we find that the radiation efficiencies in the star capture processes by a black hole $(\mathrm{BH})$ are also very different. This may provide a useful way to distinguish the capture of MS and $\mathrm{G}$ stars. Comparing with observations of the very high energy (VHE) gamma-ray emissions, we argue the event that triggers the gamma-ray emission in the energy range 4-40 TeV should be a G star capture. On the other hand, the capture of MS stars by the massive BH is required when the measured spectrum of VHE gamma-rays extends from $10^{9}$ to $10^{15} \mathrm{eV}$.
\end{abstract}

Keywords. Galaxy: center - galaxies: active - galaxies: jets - galaxies: gamma rays - accretion, accretion disks - black holes

When a star with a given mass of $M_{*}$ and radius of $R_{*}$ passes by a $\mathrm{BH}$ with a mass of $M_{b h}$, the star could be tidally disrupted and captured. This picture is described in the left panel of Fig.1. In the phase 2 (the fallback stage), a fraction of the material in the disrupted stars remains gravitationally bound to the $\mathrm{BH}$ at the pericenter. The timescale and the accretion rate (Phinney 1989) for the debris to return to the pericenter are $t_{m i n} \approx 0.11 m_{*}^{-1} r_{*}^{3 / 2} M_{6}^{1 / 2} y r$ and $\dot{M}=1.29 f_{0.3} m_{*}^{2} r_{*}^{-3 / 2} M_{6}^{-1 / 2}\left(\frac{t}{t_{m i n}}\right)^{-5 / 3} M_{\odot} y r^{-1}$, respectively, where $m_{*}=M_{*} / M_{\odot}, M_{6}=M_{b h} / 10^{6} M_{\odot}, r_{*}=R_{*} / R_{\odot}, f$ is the fraction of the stellar material falling back to the periastron, and $f_{0.3}=f / 0.3$. Assuming that the fallback material radiates the energy release promptly, the radiation efficiency $\epsilon$ (Li et al. $2002)$ is $\epsilon \approx 5.38 \times 10^{-3} r_{*}^{-1} m_{*}^{1 / 3} M_{6}^{2 / 3}$. Immediately, the total kinetic energy of the jet can be estimated as ( $\mathrm{Lu}$ et al. 2006), $E_{j \text { tot }}^{\text {proton }}=\int_{t_{\text {peak }}}^{t_{\text {crit }}} q_{j} \epsilon \dot{M} c^{2} d t$, where $q_{j}$ is the efficiency that transfers the accretion energy into the jet power, $t_{\text {peak }} \sim 0.157 m_{*}^{-1} r_{*}^{3 / 2} M_{6}^{1 / 2} y r$ and $t_{\text {crit }} \sim 1.67 \times 10^{2} f_{0.3}^{3 / 5} m_{*}^{2 / 5} M_{6}^{3 / 5} \mathrm{yr}$ are the time since the radiation reaches the peak luminosity and the time that the jet exists, respectively. The viscous accreting timescale (Lu et al. 2006) is $t_{a c c} \approx 2.08 \times 10^{2}(h / r)_{-2}^{-2} \alpha_{0.1}^{-1} m_{*}^{-1 / 2} r_{*}^{3 / 2} y r$, where $(h / r)_{-2}=(h / r) / 10^{-2}$ and $\alpha_{0.1}=\alpha / 0.1$. For typical MS stars with masses $0.08 M_{\odot} \leqslant M_{*} \leqslant 1 M_{\odot}$, the radius and mass could be related by $r_{*}=m_{*}$. And for $\mathrm{G}$ stars, the radius-mass relation (Joss et al. 1987) is $r_{*} \approx \frac{3.7 \times 10^{3} m_{*}^{4}}{1+m_{*}^{3}+1.75 m_{*}^{4}}$, where $m_{*} \equiv M_{c} / M_{\odot}$ for a giant star, $M_{c}$ is a mass of its core, and $0.17 M_{\odot} \lesssim M_{c} \lesssim 0.45 M_{\odot}$.

With these mass-radius relations of stars and $q_{j}=0.1$, the radiation efficiency and the total injection energy of protons captured by the $\mathrm{BH}$ with mass of $10^{6} M_{\odot}, 10^{7} M_{\odot}$ and $10^{8} M_{\odot}$ are plotted in Fig. 2. Paying attention to the injection energy required by the hadronic model(Aharonian \& Neronov 2005 ) to produce the VHE gamma-rays ( $\mathrm{Lu}$ et al. 2006), one can find that the $\mathrm{G}$ star capture by the $\mathrm{BH}$ is favored for the 

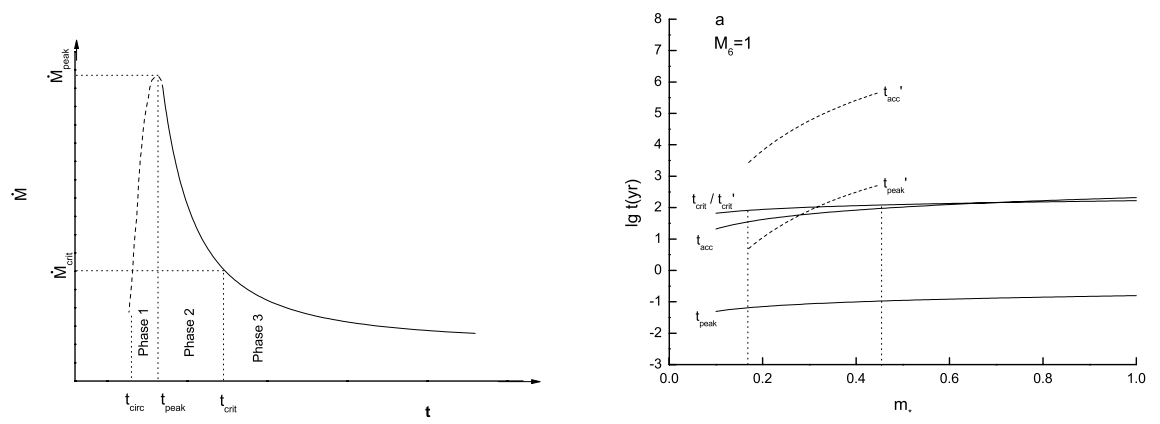

Figure 1. The left panel shows the behavior of the accretion rate versus time $(\dot{M}-t)$ when a black hole captures a star, where $t_{c i r c}=2 t_{\text {min }}$. The right panel shows the various timescales involved in the capture processes change as function of the stellar mass: $t_{a c c}, t_{\text {peak }}$ and $t_{c r i t}$ are the capture of the MS star, while those primes are for the capture of the G star.
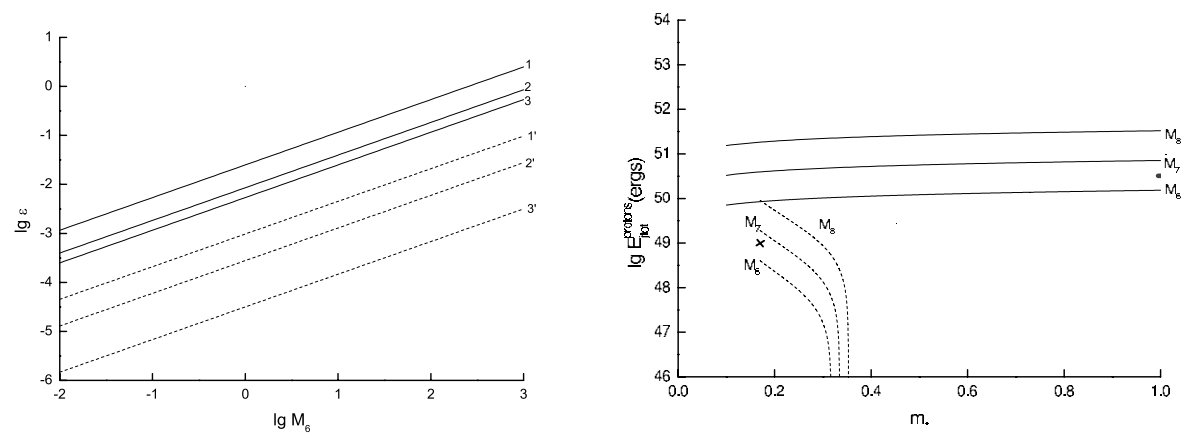

Figure 2. Lines of 1, 2, 3 in the left panel and the light lines in the right panel correspond to the capture of the MS stars, respectively. While lines of $1,2,3$, in the left panel and the black lines in the right panel are for the capture of $\mathrm{G}$ stars. In the right panel, the cross represents the capture of a $G$ star fit to the injection energy of protons required by the hadronic model with the parameters of $M_{6}=3, \epsilon=2.17 \times 10^{-3}$ and $m_{*}=0.17$; while the filled dot stands for the capture of a MS star fitting with $M_{6}=3, \epsilon=1.12 \times 10^{-2}$ and $m_{*}=1$.

gamma-rays emitted in the energy range of 4-40 TeV, and the MS case is reliable for the VHE gamma-rays with the radiated energy extended to $10^{15} \mathrm{TeV}$.

\section{Acknowledgements}

Supported by the National Natural Science Foundation of China (Grants 10273011, 10573021, 10433010), and by the Special Funds for Major State Basic Research Projects.

\section{References}

Aharonian, F. A. \& Neronov, A. 2005, ApJ, 619, 306

Joss, P. C., Rappaport, S. \& Lewis, W. 1987, ApJ, 319, 180

Li, L. X., Narayan, R. \& Menou, K. 2002, ApJ, 576, 753

Lu, Y., Cheng, K. S. \& Huang, Y. F. 2006, ApJ, 641, 288

Phinney, E. S. 1989, Nature, 340, 595 